\title{
Characterization of MR Imaging-Visible Perivascular Spaces in the White Matter of Healthy Adolescents at 3T
}

\author{
(D). Piantino, (DE.L. Boespflug, (D)D.L. Schwartz, (D) M. Luther, (D) A.M. Morales, (D)A. Lin,
} (D) R.V. Fossen, D. Silbert, and (D)B.J. Nagel

\begin{abstract}
BACKGROUND AND PURPOSE: Perivascular spaces play a role in cerebral waste removal and neuroinflammation. Our aim was to provide data regarding the burden of MR imaging-visible perivascular spaces in white matter in healthy adolescents using an automated segmentation method and to establish relationships between common demographic characteristics and perivascular space burden.
\end{abstract}

MATERIALS AND METHODS: One hundred eighteen 12- to 21-year-old subjects underwent T1- and T2-weighted 3T MR imaging as part of the National Consortium on Alcohol and Neurodevelopment in Adolescence. Perivascular spaces were identified in WM on T2-weighted imaging using a local heterogeneity approach coupled with morphologic constraints, and their spatial distribution and geometric characteristics were assessed.

RESULTS: MR imaging-visible perivascular spaces were identified in all subjects (range, 16-287). Males had a significantly higher number of perivascular spaces than females: males, mean, $98.4 \pm 50.5$, versus females, $70.7 \pm 36.1$, $(P<.01)$. Perivascular space burden was bilaterally symmetric $(r>0.4, P<.01)$, and perivascular spaces were more common in the frontal and parietal lobes than in the temporal and occipital lobes $(P<.01)$. Age and pubertal status were not significantly associated with perivascular space burden.

CONCLUSIONS: Despite a wide range of burden, perivascular spaces are present in all healthy adolescents. Perivascular space burden is higher in adolescent males than in females, regardless of age and pubertal status. In this population, perivascular spaces are highly symmetric. Although widely reported as a feature of the aging brain, awareness of the presence of perivascular spaces in a cohort of healthy adolescents provides the foundation for further research regarding the role of these structural variants in health and disease.

ABBREVIATIONS: $\mathrm{CF}=$ count fraction; NCANDA = National Consortium on Alcohol and Neurodevelopment in Adolescence; PVS = perivascular space; $\mathrm{VF}=$ volume fraction

A recent resurgence in interest in the perivascular space (PVS) in the brain, particularly as a route for waste clearance, has primarily focused on its relationship with neurodegenerative diseases marked by waste accumulation. Such disorders include Alzheimer disease, cerebral amyloid angiopathy, cerebrovascular or small-vessel disease, neuroinflammation (eg, multiple sclerosis), or acute insult, as in traumatic brain injury. ${ }^{1-4}$ Perhaps due to the positive correlation between MR imaging-visible PVS burden and senescence (PVS burden remains static during childhood

Received May 19, 2020; accepted after revision July 17.

From the Department of Pediatrics (J.P., M.L.), Division of Child Neurology, Doernbecher Children's Hospital; Department of Neurology (E.L.B., D.L.S., L.S.), Layton Aging and Alzheimer's Disease Center; Advanced Imaging Research Center (D.L.S.); Departments of Psychiatry (A.M.M., R.V.F., B.J.N.), and Emergency Medicine (A.L.), Center for Policy and Research in Emergency Medicine; Oregon Health \& Science University, Portland, Oregon; and Department of Neurology (L.S.), Portland Veterans Affairs Medical Center, Portland, Oregon.

This work was supported by the National Heart, Lung, and Blood Institute, K23HL150217-01 and K12HL133115; the National Institute on Aging, K01AG059842 and P30 AG008017; and the National Institute on Drug Abuse, K01DA046649. and adolescence $\left.{ }^{5,6}\right)$, information regarding the pediatric prevalence of these spaces is sparse and generally focused on groups of clinical import, such as those with headache and migraines, developmental delay, autism, and epilepsy. ${ }^{5-10}$ However, abnormal findings of very large tumefactive and/or numerous PVSs are consistently reported in rarer pediatric diseases, such as adrenoleukodystrophy and mucopolysaccharidoses. ${ }^{11}$ There are only 2 studies of the prevalence of MR imaging-visible PVSs in large pediatric populations: Two reported that approximately $3 \%-4 \%$ of

Please address correspondence to Juan A. Piantino, MD, 707 SW Gaines St., Portland, OR 97239; e-mail: piantino@ohsu.edu

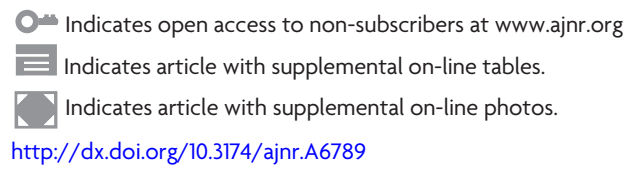


subjects presented with visible PVSs, and one reported that dilated PVSs were visible in $64 \%$. ${ }^{5,6}$ This wide variability is likely due to the MR imaging instruments used to image PVSs in vivo, historically variable definitions of "normal" and "dilated" PVSs, and inconsistent methods of quantifying visible PVS burden.

It is possible that these spaces may be visible in many more youth than previously thought; that they are almost entirely a phenomenon of the aging brain is unlikely. Perhaps due to these many factors, little is known about the timing of MR imaging-visible PVS appearance and its relation to age, pubertal status, sex differences, or spatial distribution in childhood and adolescence. Finally, a normative benchmark for the spatial distribution of these spaces may be particularly important because left-right symmetry in MR imaging-visible white matter PVSs has been broadly reported at all levels of PVS burden; frank asymmetry, along with PVS size, has been noted as clinically relevant. ${ }^{12-14}$

The primary objective of this study was to update existing literature describing normative data regarding MR imaging-visible PVS burden in white matter, including the number, volume fraction, and morphology, in a well-characterized cohort of healthy adolescents using high-field 3T MR imaging with T2-weighted imaging and manually inspected results from a fully automated segmentation algorithm. ${ }^{15}$ The secondary objective was to examine the relationship between demographic variables such as age, sex, and pubertal status on the number and morphology of PVSs in these subjects. Finally, the prevalence and degree of asymmetry was assessed and reported. These data will provide clinicians and researchers with a survey of normative data in a well-characterized clinically healthy adolescent population. This report aims to be an essential informant for future studies aimed at understanding the potential role and etiology of MR imaging-visible WM PVSs in pediatric health and disease.

\section{MATERIALS AND METHODS Study Design}

The subjects included in this study represent a subset of the cohort enrolled in the National Consortium on Alcohol and Neurodevelopment in Adolescence (NCANDA). NCANDA is a multisite, cohort-sequential, longitudinal neuroimaging study of adolescents. ${ }^{16}$ All NCANDA subjects participated in an informed-consent process. The institutional review board at Oregon Health \& Science University approved the use of de-identified data for this study.

\section{Participants}

Subjects recruited for NCANDA at a single site $(n=148)$ were eligible for inclusion in this study. Adolescents (12-21 years of age) were eligible to participate. Exclusion criteria were the following: inability to understand English; residence of $>50$ miles from the assessment site; MR imaging contraindications; current use of medications affecting brain function or blood flow (eg, antidepressants, stimulants); history of a serious medical problem that could affect MR imaging (eg, diabetes, recurrent migraine, traumatic brain injury with loss of consciousness for $>30$ minutes); mother who drank $>2$ drinks in a week or used nicotine $>10$ times per week, marijuana $>2$ times per week, or other drugs during pregnancy; prematurity ( $<30$ weeks' gestation); low birth weight or other perinatal complications requiring intervention; a current diagnosis of an Axis I psychiatric disorder (including psychosis) that would interfere with valid completion of the protocol (mild depression, simple phobia, social phobia, and attention deficit/hyperactivity disorder are not exclusionary); substance dependence; history of learning disorders; and pervasive developmental disorder or any other condition requiring specialized education. Thirty subjects with any history of injury to the head or neck-even those with no loss of consciousness-were further excluded, and the remaining 118 subjects made up the final cohort for this study. Demographic variables included sex, age, height, weight, and ethnicity. Pubertal stage was characterized using the self-assessment Pubertal Development Scale. ${ }^{17}$ The Pubertal Development Scale categories were dichotomized into early-mid pubertal and late-post pubertal.

\section{MR Imaging Acquisition and Review}

A 3D T1-weighted magnetization prepared rapid acquisition of gradient echo sequence $(\mathrm{TR}=1900 \mathrm{~ms}, \mathrm{TE}=2.92 \mathrm{~ms}, \mathrm{TI}=900 \mathrm{~ms}$, flip angle $=9^{\circ}$, imaging matrix $=256 \times 256$, FOV $=24 \mathrm{~cm}$, voxel dimensions $=1.2 \times .9375 \times 0.9375 \mathrm{~mm}, 160$ contiguous slices $)$ and a 3D T2-weighted turbo spin-echo with variable excitation pulse sequence $(\mathrm{TR} / \mathrm{TE}=3200 / 404 \mathrm{~ms}$, imaging matrix $=256 \times 256$, $\mathrm{FOV}=24 \times 24 \mathrm{~cm}$, voxel dimensions $=1.2 \times .9375 \times 0.9375 \mathrm{~mm}$, 160 contiguous slices) were performed. MR imaging volumes were collected in the sagittal plane on a 3T Tim Trio scanner (Siemens) equipped with a 12-channel head coil. T1-weighted MR images were read by a board-certified neuroradiologist. ${ }^{18}$ These reports were manually reviewed, and information about incidental findings was noted.

\section{Imaging Preprocessing}

T1-weighted images were segmented into tissue types using FreeSurfer (Version 5.1; http://surfer.nmr.mgh.harvard.edu) to yield masks of white matter, cortical gray matter, subcortical gray matter, and ventricular CSF. FreeSurfer-generated parcellations were also used to create a mask that included the cerebellum, basal ganglia, and midbrain. The T1-weighted volume was registered to the T2-weighted volume (FMRIB Linear Image Registration Tool, FLIRT; http://www.fmrib.ox.ac.uk/fsl/ fslwiki/FLIRT), and the same transformation matrix was applied to the T1-derived FreeSurfer masks described above. T2-weighted images were first up-sampled to $0.6-\mathrm{mm}$ isotropic voxels (3dresample, Analysis of Functional Neuro Images [AFNI]; http://afni.nimh.nih.gov/afni), skull-stripped (FSL Brain Extraction Tool; http://fsl.fmrib.ox.ac.uk/fsl/fslwiki/BET), and submitted to a 5 class K-means-based tissue segmentation in SPM5 (https://www.fil.ion.ucl.ac.uk/spm/doc/), which produces a homogenized image in preprocessing (Fig $1 B$ ). The tissue class corresponding to WM was thresholded at $>50 \%$ likelihood (3dcalc, AFNI; Fig 1C), holes in the resulting mask were filled (Fig $1 D$ ), and the entire mask was eroded by 2 voxels (3dmask_tool, AFNI; Fig 1E) to avoid the possibility of mis-segmented parts of neocortical gray matter being misidentified as PVS in WM. Finally, any voxels that shared membership between the resultant WM mask and the cerebellar/basal ganglia/midbrain mask were eliminated from the WM mask (3dcalc). 


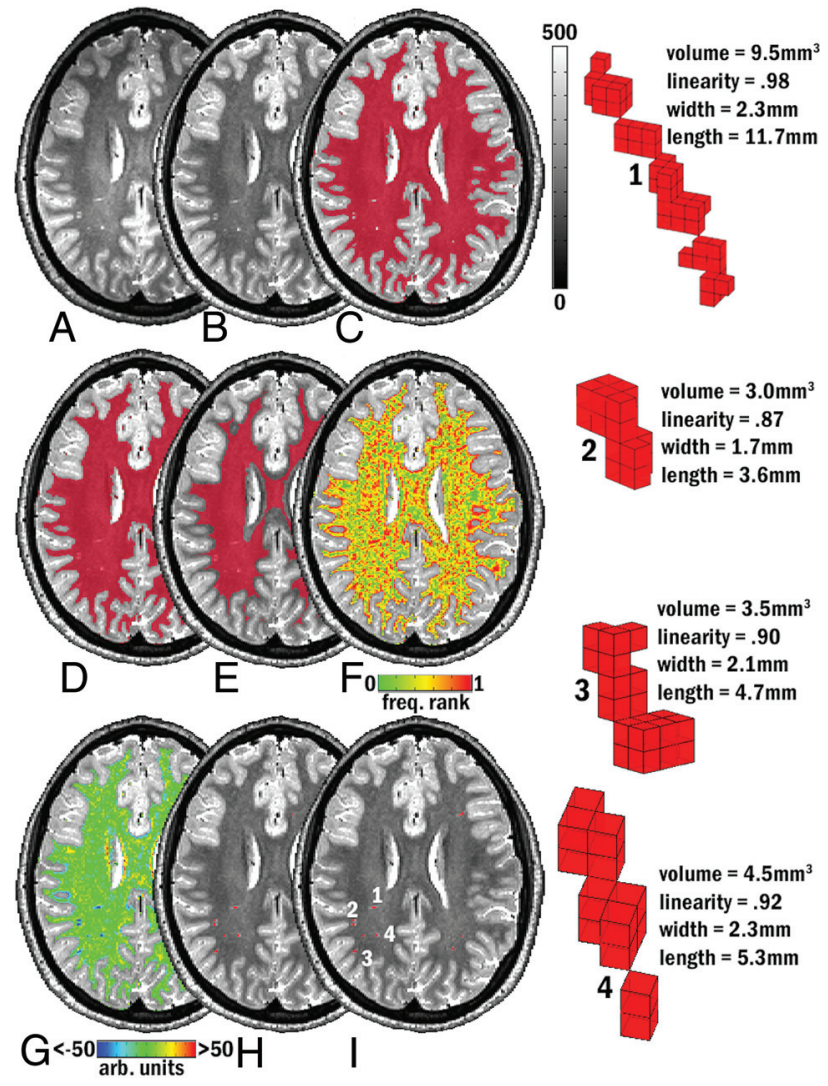

FIG 1. Examples of MR imaging-visible PVSs on T2-based images and PVS segmentation method. A, Acquired T2. B, After intensity normalization. $\mathrm{C}-\mathrm{H}$, Intermediate steps in the algorithm. $\mathrm{C}$, WM tissue mask with holes filled $(D)$ and eroded by 2 voxels $(E)$. $F$, Frequency rank map. G, Map of local-intensity difference. $H$, Segmented objects submitted to morphologic constraint. I, Final segmentation mask. 1-4, Morphologic characteristics of 4 of the final segmented PVSs visible on this axial section (see I).

\section{PVS Segmentation}

A similar methodology to that used in a previous study was used for PVS identification (Fig 1). ${ }^{15}$ Preprocessed homogenized T2weighted images were subjected to a 2-part, local heterogeneity assessment. The sphere of a radius of $3 \mathrm{~mm}$ was constructed around each voxel within the eroded WM mask, and the voxels within that sphere were ranked-based on their intensity (ie, a voxel was marked as 0.5 if its intensity fell into the 50th percentile of surrounding voxels, Fig $1 F$ ). Next, the sphere of a radius of $4 \mathrm{~mm}$ was constructed around a voxel, and the average of the difference in intensity between that voxel and surrounding voxels was calculated and that voxel was marked with the result (3dLocalstat, AFNI; Fig $1 G)$. Finally, if a voxel did not reside in the cerebellum/basal ganglia/midbrain mask, did reside in the eroded WM mask, had an average difference in intensity from surrounding voxels that was $>15 \%$ of its own intensity, and had an intensity that fell into the top fifth percentile of its neighbors, it was considered for morphologic analysis (3dcalc, AFNI; Fig $1 H)$. All clusters of $>1 \mathrm{~mm}^{3}(\sim 5$ voxels, 3D corner-to-corner connectivity, 3dclust type 3, AFNI) were passed to the morphology constraint. Linearity, width, length, and total volume of each cluster were calculated in Matlab (MathWorks) (Fig $1 I$ and Fig 1, parts 1-4); a cluster was retained as a putative PVS if the segmented object was $<16.41$-mm wide and had a linearity of at least 0.8 . Each PVS detected by the automated algorithm was assessed by a trained researcher (M.L.); false alarms were removed from further analysis. See the RESULTS section for a summary of error counts and rates.

\section{Spatial Distribution of PVS}

Delineation of WM into lobar regions (left and right frontal, temporal, parietal, and occipital) was accomplished, in part, using standard ROI output from FreeSurfer via recon-all (e.g., frontal_R_GM.nii.gz and frontal_L_GM.nii.gz). Periventricular and other deep WM regions are not marked by FreeSurfer; however, some WM PVSs exist in these unmarked WM regions, so it is necessary to classify unmarked voxels into lobes. Every voxel in the total WM mask that was not marked by FreeSurfer as belonging to juxtacortical WM was subjected to the following procedure: An integer-based (1-8, describing the 8 juxtacortical WM lobar regions above) histogram was constructed for every voxel that fell within the sphere of a radius of $7.5 \mathrm{~mm}$ from each unmarked voxel. After dividing the count of each juxtacortical WM region (1-8) by the volume of that region to account for differently sized lobes (eg, frontal $\gg$ occipital), we marked each voxel as a member of the lobe and hemisphere of the maximum normalized frequency of the histogram by a winnertake-all method (On-line Fig 1). This process failed at the FreeSurfer WM parcellation stage for 3/118 subjects ( 2 females and 1 male); all WM lobar PVS distributions are described in the remaining 115 subjects. Probability maps of PVS incidence in this cohort were generated using nonlinear registrations. All subjects' skull-stripped and homogenized T2-weighted images were affine-registered to Montreal Neurological Institute space (FLIRT, FSL, and 3dAllineate, AFNI; mni_icbm152_t2_relx_tal_nlin_sym_09a.nii.gz was used as an initial template). The resulting datasets were simultaneously submitted to template construction (antsMultivariateTemplateConstruction.sh, Advanced Normalization Tools software package [ANTS], affine + 2-step nonlinear transform, https:/github.com/ANTsX/ANTs/ issues/553). The initial affine transformation and all subsequent transformations were combined and applied to single-subject native space PVS masks with false alarms removed (antsApplyTransforms; ANTS). The mean of all transformed PVS masks was calculated; the result was voxelwise-thresholded at $0.5 \%$ likelihood and clusterthresholded at $43.2 \mathrm{~mm}^{3}$ (two hundred $0.6-\mathrm{mm}$ isotropic voxels) for ease of viewing.

\section{Statistical Analysis}

Statistical analyses were performed using STATA/MP 15 (StataCorp). Descriptive statistics were used to analyze the demographic characteristics of the cohort. Sex regression models were implemented with the total PVS number and volume as outcomes of interest; sex and age differences were analyzed using 2-sample $t$ tests. Multivariate linear regression models were implemented with total PVS number and volume as outcomes of interest; sex, age, and pubertal stage were used as covariates. PVS volume fraction (VF) and count fraction (CF) were calculated as the total volume of PVSs (cubic millimeters) or PVS count within the volume of the WM search field (cubic centimeters), respectively. The interaction of sex and age on PVS characteristics was tested with linear regression models. A 
secondary analysis included multiple linear regression with cluster characteristics (width, length, and volume) as individual outcomes and the same covariates indicated above. Differences in PVS number and volume across lobes were obtained using Pearson regression and paired and unpaired t-tests, as appropriate. An asymmetry index was created by calculating the absolute value of the log of the left divided by the right CF and VF so that departure from zero (ie, symmetric) indicated asymmetry; subjects were identified as outliers if asymmetry indices were $\geq 2.5$ SDs from the population mean. Regression diagnostics for all models were examined for model fit. All reported $P$ values are 2-sided, and the threshold for statistical significance set to an $\alpha$ of .05.

\section{Data Availability Statement}

The data that support the findings of this study are available from the NCANDA study on request. The statistical code used to analyze these data is available on request.

\section{RESULTS}

Table 1 provides demographic information and characteristics of the study cohort. Fifty-six subjects in the cohort (47\%) were male. The median age was 16 years (interquartile range, $14.1-18.3$ years) for males, and 16.7 years (interquartile range, 14.3-18.4 years) for females. In order to study a diverse cohort, both Hispanic and Non-Hispanic subjects were included. Seven subjects in the cohort (6\%) identified themselves as Hispanic. Nine subjects had incidental intracranial findings noted by a neuroradiologist (3 instances of mega cisterna magna; 2 pineal cysts; 1 tonsillar ectopia; 1 gray matter heterotopia; 1 large vein; 1 large prepontine cistern). None of the subjects had prominent perivascular spaces noted by a neuroradiologist. After adjusting for age, the male brain volume was

\section{Table 1: Cohort characteristics}

\begin{tabular}{lcc}
\hline Patient Characteristics (Median) (IQR) & Males $(\boldsymbol{n}=\mathbf{5 6})$ & Females $(\boldsymbol{n}=\mathbf{6 2})$ \\
\hline Age $(\mathrm{yr})$ & $16(14.1-18.3)$ & $16.7(14.3-18.4)$ \\
Weight (kg) & $63.5(56.2-81.6)$ & $57.6(52.1-63.5)$ \\
Height $(\mathrm{cm})$ & $169(163.8-180.3)$ & $162.5(157.4-167.6)$ \\
BMI (kg/m $\left.{ }^{2}\right)$ & $22(19.3-26.1)$ & $21.8(19.5-24)$ \\
PDS score & $3.1(2.6-3.6)$ & $3.6(2.6-3.6)$ \\
Ethnicity (No.) (\%) & & \\
$\quad$ Hispanic & $5(9)$ & $2(3)$ \\
Non-Hispanic & $51(91)$ & $60(97)$ \\
\hline
\end{tabular}

Note:-BMI indicates body mass index; PDS, Pubertal Development Scale; IQR, interquartile range.
$145.2 \mathrm{~cm}^{3}$ larger than that of females $\left(95 \% \mathrm{CI}, 108.5-182 \mathrm{~cm}^{3}\right.$; $P<.01)$, and male white matter volume was $58.5 \mathrm{~cm}^{3}$ larger than that of females ( $95 \% \mathrm{CI}, 41-76 \mathrm{~cm}^{3} ; P<.01$, On-line Table 1 ). In this cohort, age was not significantly associated with brain volume or total white matter volume (On-line Fig 1). Plots of residuals versus predicted values, QQ plots, partial regression plots, and outlier and leverage statistics indicated a good model fit. The total error rate (of 12,274 detected objects) of the PVS segmentation algorithm in this dataset was $19.4 \%$ (2372). Most false alarms were attributable to hypointense WM voxels other than PVS (1696 or $13.8 \%)$. The remaining false alarms were attributable to partial inclusion of GM in the basal ganglia (676 or 5.5\%) (On-line Fig 3).

\section{PVS Characteristics}

Examples of MR imaging-visible PVSs detected by the segmentation algorithm can be seen in Fig 1I. PVSs were observed in the supratentorial white matter of all 118 subjects in the cohort (Table 2). Males had a significantly higher absolute number of PVSs than females (mean, $98.4 \pm 50.5$ and $70.7 \pm 36.1$, respectively; $P<.01$ ). Males also had a higher total volume of PVSs than females (mean, $334.8 \pm 192.4 \mathrm{~mm}^{3}$ and $241.2 \pm 134 \mathrm{~mm}^{3}$, respectively; $P<.01$ ). After adjusting for white matter volume, males had higher PVS number per cubic centimeter of white matter than females (CF mean, $0.2 \pm 0.1$ and $0.1 \pm 0.07$, respectively; $P=.02$ ). Males also had a higher PVS volume per cubic centimeter of white matter than females (VF mean, $0.7 \pm 0.4$ and $0.5 \pm 0.3$, respectively; $P=.04$ ). The morphologic characteristics of PVS (ie, length, width, and volume) were not statistically different between males and females $(P>.35)$. After we adjusted for sex, no effect of age or pubertal stage was observed on total PVS volume, total PVS count, and PVS VF or CF ( $P>.3$, On-line Table 2 and On-line Fig 4). There were no significant interactions between age and sex on any of the above-mentioned PVS characteristics $(P=.68-.94)$.

\section{PVS Location by Lobe}

PVS volume, count, VF, and CF were significantly positively correlated across hemispheres in each lobe (Pearson $r>0.4, P<.01$, Fig 2). Significant asymmetry in this population was defined as 1 hemisphere having approximately $225 \%$

Table 2: Characteristics of PVS identified with the automated algorithm

\begin{tabular}{|c|c|c|c|c|c|}
\hline \multirow[b]{2}{*}{ Characteristic } & \multirow[b]{2}{*}{$P$ Value $^{\mathrm{a}}$} & \multicolumn{2}{|c|}{ Females $(n=62)$} & \multicolumn{2}{|c|}{ Males $(n=56)$} \\
\hline & & Mean (SD) & Range & Mean (SD) & Range \\
\hline \multicolumn{6}{|l|}{ PVS whole brain } \\
\hline PVS total No. & $<.01$ & $70.7(36.1)$ & 20-153 & $98.4(50.5)$ & $16-287$ \\
\hline PVS total volume $\left(\mathrm{mm}^{3}\right)$ & $<.01$ & $241.2(134)$ & $74.7-617$ & $334.8(192.4)$ & $43-1103.3$ \\
\hline PVS WM (No. $\left./ \mathrm{cm}^{3}\right)$ & .02 & $0.1(0.07)$ & $0.04-0.36$ & $0.2(0.1)$ & $0.04-0.6$ \\
\hline PVS volume WM $\left(\mathrm{mm}^{3} / \mathrm{cm}^{3}\right)$ & .04 & $0.5(0.3)$ & $0.16-1.3$ & $0.7(0.4)$ & $0.1-2.3$ \\
\hline \multicolumn{6}{|l|}{ Individual PVS cluster } \\
\hline Individual cluster length (mm) & .75 & $3.6(0.2)$ & $3.3-4.5$ & $3.6(0.2)$ & $3-4.3$ \\
\hline Individual cluster width (mm) & .35 & $2(0.07)$ & $1.8-2$ & $2(0.08)$ & $1.7-2$ \\
\hline Individual cluster volume $\left(\mathrm{mm}^{3}\right)$ & .80 & $2.9(0.3)$ & $2.1-3.8$ & $2.8(0.3)$ & $2-3.6$ \\
\hline Cluster volume $\left(\mathrm{mm}^{3}\right)$ by total cluster (No.) & .80 & $0.05(0.02)$ & $0.02-0.16$ & $0.03(0.02)$ & $0.01-0.14$ \\
\hline
\end{tabular}

\footnotetext{
${ }^{\mathrm{a}}$ Two-sample $t$ test.
} 


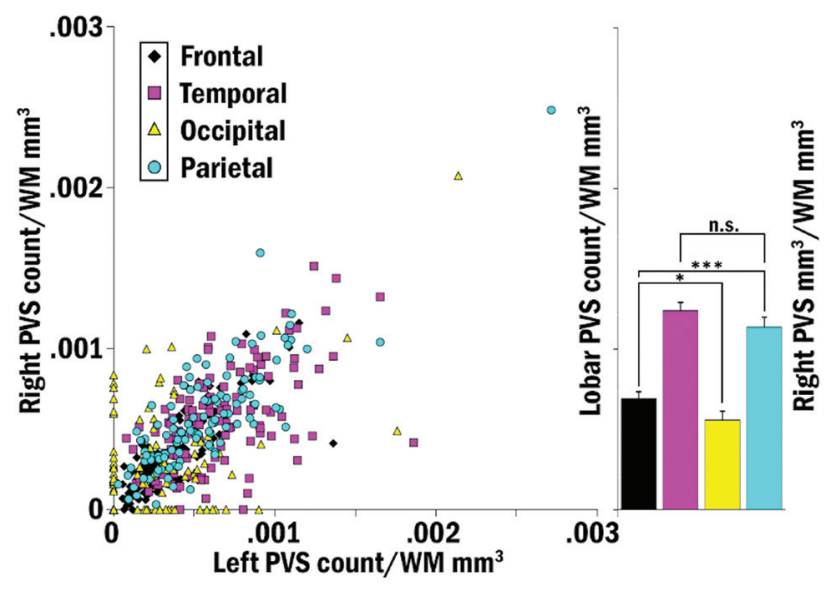

FIG 2. PVS burden in the right and left hemispheres. PVS count and white matter volume are correlated between hemispheres in all lobes. Barplots illustrate significantly different PVS count/white matter volume by lobe across hemispheres. Asterisk indicates $P<.05$; triple asterisks, $P<.001$.
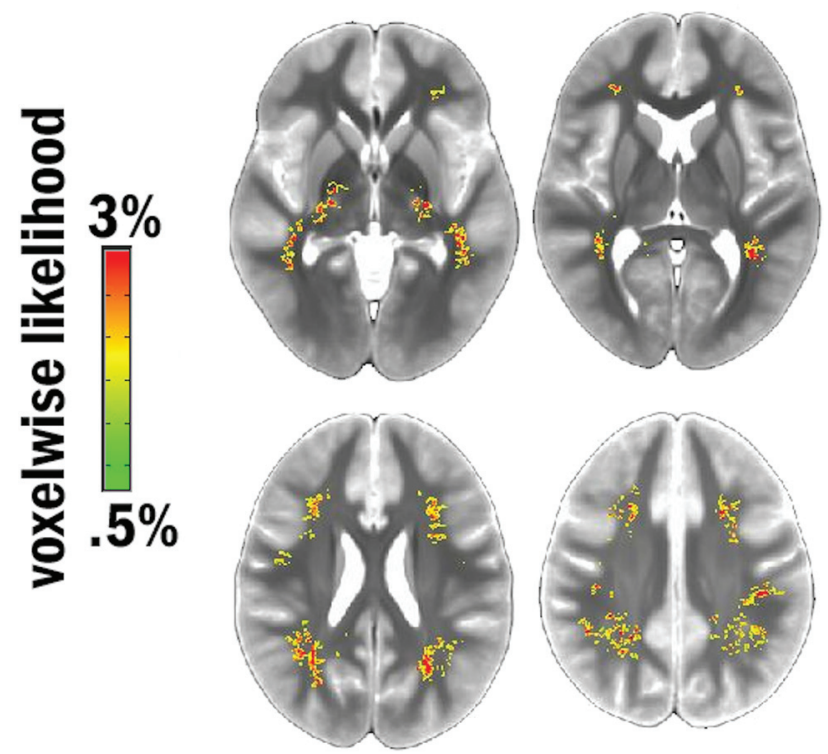

FIG 3. Map of the likelihood of a segmented PVS at a given voxel across all subjects.

of the burden of the other hemisphere (asymmetry index $\approx 0.34$, approximately $2.5 \mathrm{SDs}$ above the mean). One male and 2 females (1.7\% and $3.7 \%)$ had asymmetry indices for VF that exceeded this cutoff; CF asymmetry was similarly distributed across the population (2 [3.3\%] and 2 [3.7\%] for males and females, respectively). There was no difference in asymmetry indices between males and females (VF or $\mathrm{CF}, P>.1$ ) nor was there an association between $\mathrm{CF}$ or VF asymmetry and age $(P>.1)$. Over both hemispheres, PVS CF was significantly higher in the parietal and temporal lobes than the frontal lobe; the frontal lobe bore a significantly higher PVS CF than the occipital lobe (all $P<.01$, bar graphs in Fig 2). The same relationships were true of PVS VF. There were more PVSs in the parietal than frontal lobes, more in frontal than temporal lobes, and more in temporal than occipital lobes; the same relationships were true of lobar PVS volume (all $P<.001$, data not shown). PVS CF was also compared within lobes between sexes (male $>$ female, $P<.05$ in the temporal lobe; all other lobes were nonsignificant) and across sexes within lobes between hemispheres (the burden was higher in the left temporal lobe than in the right, $P<.01$; On-line Fig 5, center column). The same relationships were observed for VF (data not shown). A brain-wide map of voxelwise probability for a detected PVS can be found in Fig 3.

\section{DISCUSSION}

In this study, we quantify the number and morphology of white matter PVSs in a cohort of healthy adolescents under rigorous study-entry criteria. Using $3 \mathrm{~T}$ MR imaging scans and objective quantitative methods, we demonstrate that PVSs are seen in this population in a much larger proportion of subjects than previously described in the literature. ${ }^{5,6}$ We provide normative PVS morphologic characteristics, including total number, volume, and individual length, width, and diameter of PVSs in this age group and the distribution of lobar locations of PVS burden. We show that male adolescents have a higher total number and volume of PVSs per unit of white matter than females. We show that in adolescents, individual PVS morphology (length, width, and diameter) is not associated with age, sex, or increasing white matter volume. Finally, we report the incidence rate of asymmetric burden. These findings support the notion that PVSs are seen in MRIs of healthy individuals.

In adults, the presence of PVSs has been mainly linked to the aging process ${ }^{9}$ and to vascular and Alzheimer dementias. ${ }^{19,20}$ Two studies in youth concluded that in this population, PVSs were either absent or a rare finding, present in $<5 \%$ of subjects. ${ }^{5,6}$ These early studies, although considered the first attempts at quantifying PVS in children, have limitations. Counts were performed on MRIs obtained using a $1.5 \mathrm{~T}$ field strength, introducing a floor effect for small structures with limited contrast to surrounding tissue due to partial volume effects. Early studies used subjective visual ratings, which are subject to interrater variability and differences in interpretation and do not provide morphologic details about each individual PVS. Although children were labeled as "healthy," MRIs were performed in subjects with entities such as benign epilepsy, headaches, or behavioral problems, which limit their generalizability. ${ }^{5}$ Early studies did not adjust for other important covariates such as sex or total cerebral white matter. The results presented here expand this previous work by filling existing gaps in the limited literature addressing this phenomenon. NCANDA participants met rigorous study entry criteria that provided us with important baseline clinical and demographic information on each subject. NCANDA participants underwent 3T MR imaging, which provided adequate visualization of PVSs. By incorporating objective PVS quantification, we minimized visual rater irregularities. We were also able to provide details about the morphology of each PVS, including important characteristics such as width, length, and volume.

In this cohort, males had a higher number of morphologically similar PVSs than females. Most important, age and pubertal stage were not significantly associated with PVS number or total volume. Furthermore, there was no interaction between age and sex on PVS burden. A higher prevalence of "large" ( $>2 \mathrm{~mm}$ in diameter) PVSs in males than in females has been reported in subjects aged 
0-99 years using a visual rating scale. ${ }^{9}$ The authors, however, did not provide an analysis of sex differences across age groups. A separate study using a volumetric approach in an adult population also identified higher burden volumes in males over females in control subjects. ${ }^{1}$ The timing and etiology of sex differences are yet to be elucidated. However, the fact that sex differences are already present in early-pubertal individuals argues in favor of a much earlier etiology than previously established. These differences may be present at birth or develop during childhood.

In agreement with previous work, PVS burden in this cohort was highest in the frontal and parietal lobes. ${ }^{5,6}$ However, PVSs were also found in the temporal and, more rarely, the occipital lobes. The appearance of PVSs in the temporal and occipital lobes could be, in part, attributed to the better resolution of the $3 \mathrm{~T}$ scans and the objective measurements used in our study, resulting in a higher sensitivity for PVS detection. In our cohort, PVS burden was bilaterally symmetric, and a high burden in 1 hemisphere, regardless of the measure used for burden or of the lobar location, is indicative of a high burden in the contralateral side. It is certain that the high likelihood of burden symmetry across hemispheres is a reflection of the symmetry of cerebral vasculature. However, the high interindividual variability in overall MR imaging-visible PVSs (approximately an 8fold difference) remains unexplained. In our cohort, pubertal status and age were not associated with PVS burden. It is possible that other variables not included in this analysis may affect PVS burden. For example, sleep efficiency has been shown to affect PVS burden in adults, and it may play a role in PVS burden variability in adolescents. ${ }^{21}$ Last, the incidence rate of a significantly asymmetric burden in healthy adolescents should be considered $1 \%-3 \%$, according to these data. This suggests that an asymmetric burden, defined as approximately $>225 \%$ of PVS count or volume between hemispheres, is a rare occurrence, though the clinical significance of such a finding remains unclear.

Finally, we observed a difference in burden per unit WM between lobes. It is unlikely that WM is perfused by nearly twice as many vessels in the temporal and parietal lobes than in the frontal and occipital lobes per unit tissue; if the interpretation of a difference in burden between lobes is predicated on an uneven distribution of lobar vasculature, then the most parsimonious explanation is that these spaces are organized about the venous system. Therefore, the finding may arise from either an uneven lobar distribution of venous architecture in WM or because the parietal and temporal lobes are more susceptible to the underlying cause of PVS expansion, or both. Given that the identity, artery or vein, of the vessels in WM around which these spaces are visible remains an open question, this report may serve to inform this debate considerably, and strongly suggests that venous return is the primary system around which these spaces expand in WM. Another possibility is that the temporal and parietal lobes contain some characteristic that makes a member voxel more likely to be a PVS; in either case, the finding provides a clue to the underlying cause of the fact that some of these spaces are large enough to view on a standard clinical MR imaging instrument and some are not.

Previous studies have found no correlation between the number of PVSs and age in young individuals. ${ }^{5,6}$ While a strong relationship between MR imaging-visible PVSs and age has been noted in patients older than 60 years, ${ }^{9}$ other studies suggest that the relationship may be regionally dependent ${ }^{22}$ or dependent on the resolution of the images acquired. ${ }^{23}$ Future studies that objectively address the evolution of age- and sex-related differences in PVS burden across the life span, especially ones with longitudinal designs, are needed to further elucidate the potential clinical relevance of these findings.

This study has several limitations. The cross-sectional design of this analysis precludes the possibility of determining individual longitudinal changes in PVS burden. Future investigations, including longitudinal data from this cohort, should be conducted to better understand individual differences in the development of this phenomenon. Second, the present methodology is limited to white matter. Given evidence that PVSs do occur in other regions, including the thalamus, midbrain, cerebellum, insular cortex, extreme capsule, optic tract, and hippocampus, future studies evaluating these regions in an adolescent cohort are certainly warranted. ${ }^{24,25}$

\section{CONCLUSIONS}

Using objective measurements in a rigorously selected cohort, we conclude that PVSs are seen in clinically healthy adolescents, that males have higher PVS burden than females, and that while overall burden varies widely, burden symmetry across hemispheres is preserved. These differences are not related to age, total white matter volume, or pubertal status. These findings establish a baseline from which PVS burden can be investigated in different disease processes and present the foundation for further research in the physiology and pathology of these structures in youth.

Disclosures: Juan Piantino-RELATED: Grant: National Institutes of Health, Comments: National Heart, Lung, and Blood Institute, K23HL150217-01. Erin Boespflug - RELATED: Grant: National Institutes of Health, Comments: research salary support from National Institutes of Health grants K01AG059842 and P30AG008017.* Amber Lin—RELATED: Grant: National Institutes of Health K12 training grant, Comments: This grant supports my time (biostatistical support) for a number of investigators, including the primary author of this article. Lisa Silbert-UNRELATED: Grants/Grants Pending: National Institutes of Health, Comments: Principal Investigator on a National Institutes of Health R01 and CoInvestigator on several National Institutes of Health grants, not directly related to this article.* Bonnie J. Nagel—RELATED: Grant: National Institute on Alcohol Abuse and Alcoholism, Comments: This work was supported by $\mathrm{NIH} \cup 01$ AA02169.* *Money paid to the institution.

\section{REFERENCES}

1. Ramirez J, Berezuk C, McNeely AA, et al. Visible Virchow-Robin spaces on magnetic resonance imaging of Alzheimer's disease patients and normal elderly from the Sunnybrook Dementia Study. J Alzheimers Dis 2014;43:415-44 CrossRef Medline

2. Potter GM, Chappell FM, Morris Z, et al. Cerebral perivascular spaces visible on magnetic resonance imaging: development of a qualitative rating scale and its observer reliability. Cerebrovasc Dis 2015;39:224-31 CrossRef Medline

3. Patankar TF, Mitra D, Varma A, et al. Dilatation of the VirchowRobin space is a sensitive indicator of cerebral microvascular disease: study in elderly patients with dementia. AJNR Am J Neuroradiol 2005;26:1512-20 Medline

4. Charidimou A, Jaunmuktane Z, Baron JC, et al. White matter perivascular spaces: an MRI marker in pathology-proven cerebral amyloid angiopathy? Neurology 2014;82:57-62 CrossRef Medline

5. Groeschel S, Chong WK, Surtees R, et al. Virchow-Robin spaces on magnetic resonance images: normative data, their dilatation, and a review of the literature. Neuroradiology 2006;48:745-54 CrossRef Medline 
6. Rollins NK, Deline C, Morriss MC. Prevalence and clinical significance of dilated Virchow-Robin spaces in childhood. Radiology 1993;189:53-57 CrossRef Medline

7. Schick S, Gahleitner A, Wober-Bingol C, et al. Virchow-Robin spaces in childhood migraine. Neuroradiology 1999;41:283-87 CrossRef Medline

8. Edvinsson L, Haanes KA, Warfvinge K. Does inflammation have a role in migraine? Nat Rev Neurol 2019;15:483-90 CrossRef Medline

9. Heier LA, Bauer CJ, Schwartz L, et al. Large Virchow-Robin spaces: MRclinical correlation. AJNR Am J Neuroradiol 1989;10:929-36 Medline

10. Liu C, Habib T, Salimeen M, et al. Quantification of visible VirchowRobin spaces for detecting the functional status of the glymphatic system in children with newly diagnosed idiopathic generalized epilepsy. Seizure 2020;78:12-17 CrossRef Medline

11. Murata R, Nakajima $S$, Tanaka A, et al. MR imaging of the brain in patients with mucopolysaccharidosis. AJNR Am J Neuroradiol 1989;10:1165-70 Medline

12. Austein F, Langguth $P$, Lindner T. Extreme widening of asymmetric giant cystic Virchow-Robin spaces. Neuroradiology 2018;60:3-5 CrossRef Medline

13. Ogawa $\mathrm{T}$, Okudera $\mathrm{T}$, Fukasawa $\mathrm{H}$, et al. Unusual widening of Virchow-Robin spaces: MR appearance. AJNR Am J Neuroradiol 1995;16:1238-42 Medline

14. Sawada M, Nishi S, Hashimoto N. Unilateral appearance of markedly dilated Virchow-Robin spaces. Clin Radiol 1999;54:334-36 CrossRef Medline

15. Schwartz DL, Boespflug EL, Lahna DL, et al. Autoidentification of perivascular spaces in white matter using clinical field strength $\mathrm{T} 1$ and FLAIR MR imaging. Neuroimage 2019;202:116126 CrossRef Medline

16. Brown SA, Brumback $T$, Tomlinson $K$, et al. The National Consortium on Alcohol and NeuroDevelopment in Adolescence (NCANDA): a multisite study of adolescent development and substance use. J Stud Alcohol Drugs 2015;76:895-908 CrossRef Medline

17. Petersen AC, Crockett L, Richards M, et al. A self-report measure of pubertal status: reliability, validity, and initial norms. J Youth Adolesc 1988;17:117-33 CrossRef Medline

18. Sullivan EV, Lane B, Kwon D, et al. Structural brain anomalies in healthy adolescents in the NCANDA cohort: relation to neuropsychological test performance, sex, and ethnicity. Brain Imaging Behav 2017;11:1302-15 CrossRef Medline

19. Maclullich AM, Wardlaw JM, Ferguson KJ, et al. Enlarged perivascular spaces are associated with cognitive function in healthy elderly men. J Neurol Neurosurg Psychiatry 2004;75:1519-23 CrossRef Medline

20. Weller RO, Subash M, Preston SD, et al. Perivascular drainage of amyloid-beta peptides from the brain and its failure in cerebral amyloid angiopathy and Alzheimer's disease. Brain Pathol 2008;18:253-66 CrossRef Medline

21. Berezuk C, Ramirez J, Gao F, et al. Virchow-Robin spaces: correlations with polysomnography-derived sleep parameters. Sleep 2015;38:85358 CrossRef Medline

22. Dubost F, Yilmaz P, Adams H, et al. Enlarged perivascular spaces in brain MRI: automated quantification in four regions. Neuroimage 2019;185:534-44 CrossRef Medline

23. Zong X, Park SH, Shen D, et al. Visualization of perivascular spaces in the human brain at 7T: sequence optimization and morphology characterization. Neuroimage 2016;125:895-902 CrossRef Medline

24. Jungreis CA, Kanal E, Hirsch WL, et al. Normal perivascular spaces mimicking lacunar infarction: MR imaging. Radiology 1988;169:10104 CrossRef Medline

25. Hirabuki N, Fujita N, Fujii K, et al. MR appearance of VirchowRobin spaces along lenticulostriate arteries: spin-echo and twodimensional fast low-angle shot imaging. AJNR Am J Neuroradiol 1994;15:277-81 Medline 